\title{
Efficacy of vagus nerve stimulation in posttraumatic versus nontraumatic epilepsy
}

\author{
Clinical article
}

\author{
Dario J. Englot, M.D., Ph.D., 1,2 John D. Rolston, M.D., Ph.D., ${ }^{1,2}$ \\ Doris D. Wang, M.D., Ph.D., ${ }^{1,2}$ Kevin H. Hassnain, M.S., ${ }^{3}$ Charles M. Gordon, M.S., ${ }^{3}$ \\ and Edward F. Chang, M.D., ${ }^{1,2}$ \\ ${ }^{I}$ Comprehensive Epilepsy Center and ${ }^{2}$ Department of Neurological Surgery, University of California, San \\ Francisco, California; and ${ }^{3}$ Cyberonics, Inc., Houston, Texas
}

\begin{abstract}
Object. In the US, approximately 500,000 individuals are hospitalized yearly for traumatic brain injury (TBI), and posttraumatic epilepsy (PTE) is a common sequela of TBI. Improved treatment strategies for PTE are critically needed, as patients with the disorder are often resistant to antiepileptic medications and are poor candidates for definitive resection. Vagus nerve stimulation (VNS) is an adjunctive treatment for medically refractory epilepsy that results in $\mathrm{a} \geq 50 \%$ reduction in seizure frequency in approximately $50 \%$ of patients after 1 year of therapy. The role of VNS in PTE has been poorly studied. The aim of this study was to determine whether patients with PTE attain more favorable seizure outcomes than individuals with nontraumatic epilepsy etiologies.

Methods. Using a case-control study design, the authors retrospectively compared seizure outcomes after VNS therapy in patients with PTE versus those with nontraumatic epilepsy (non-PTE) who were part of a large prospectively collected patient registry.

Results. After VNS therapy, patients with PTE demonstrated a greater reduction in seizure frequency $(50 \%$ fewer seizures at the 3-month follow-up; $73 \%$ fewer seizures at 24 months) than patients with non-PTE (46\% fewer seizures at 3 months; $57 \%$ fewer seizures at 24 months). Overall, patients with PTE had a $78 \%$ rate of clinical response to VNS therapy at 24 months (that is, $\geq 50 \%$ reduction in seizure frequency) as compared with a $61 \%$ response rate among patients with non-PTE (OR 1.32, 95\% CI 1.07-1.61), leading to improved outcomes according to the Engel classification ( $\mathrm{p}<0.0001$, Cochran-Mantel-Haenszel statistic).

Conclusions. Vagus nerve stimulation should be considered in patients with medically refractory PTE who are not good candidates for resection. A controlled prospective trial is necessary to further examine seizure outcomes as well as neuropsychological outcomes after VNS therapy in patients with intractable PTE.

(http://thejns.org/doi/abs/10.3171/2012.8.JNS122)
\end{abstract}

\section{KeY Words • posttraumatic epilepsy • seizure • epilepsy • traumatic brain injury $\quad$ vagus nerve stimulation}

I $\mathrm{N}$ the US, approximately 500,000 individuals are hospitalized yearly for TBI. ${ }^{1,15,21}$ Posttraumatic epilepsy is a common sequela of TBI, accounting for $20 \%$ of symptomatic epilepsy cases in the general population and afflicting up to half of the military personnel who suffer penetrating head injury. ${ }^{1,4,31}$ Unfortunately, seizures in PTE are frequently resistant to antiepileptic medications, and patients with PTE are often poor candidates for definitive resection because of poor localization of a single

\footnotetext{
Abbreviations used in this paper: $\mathrm{AED}=$ antiepileptic drug; $\mathrm{PTE}$ $=$ posttraumatic epilepsy; TBI = traumatic brain injury; VNS = vagus nerve stimulation.
}

epileptic focus. ${ }^{15}$ Thus, improved treatment strategies for TBI-related epilepsy are critically needed.

The US FDA approved VNS as an adjunctive treatment for medically refractory partial epilepsy in 1997, and stimulators have now been implanted in over 60,000 patients with epilepsy worldwide. , $9,32^{6}$ The neurobiological underpinnings of VNS in epilepsy remain poorly understood, although human and animal studies have suggested that vagal stimulation may desynchronize and decrease abnormal electroencephalographic spiking patterns..$^{10,18,23}$ Overall, approximately $50 \%$ of patients with epilepsy benefit from $\geq 50 \%$ fewer seizures after 1 year of VNS therapy, although complete seizure freedom is rarely achieved. ${ }^{13,14}$ 
The potential benefits of VNS therapy in the PTE population have been poorly studied. However, 2 recent retrospective case series have revealed dramatic reductions in seizure frequency among patients with PTE $(68 \%-85 \%)$ after VNS therapy, suggesting that patients with PTE may respond more favorably to treatment than those with nontraumatic epileptic disorders. ${ }^{11,24}$ Furthermore, a recent meta-analysis has indicated that patients with PTE may achieve greater benefits from VNS than individuals with other epilepsy etiologies, but these conclusions were limited by the small number of patients in the study. ${ }^{14}$

Here, we examined a large, prospectively collected patient registry and analyzed seizure outcomes after VNS for drug-resistant epilepsy to determine whether patients with PTE attain more favorable seizure outcomes than individuals with nontraumatic epilepsy etiologies.

\section{Methods}

\section{Patient Outcome Registry Data Collection}

All data were obtained from the VNS Therapy Patient Outcome Registry maintained by the manufacturer of the VNS therapy system (Cyberonics, Inc.). This database was established in 1999, after FDA approval of VNS for the treatment of epilepsy in 1997, to systematically monitor patient outcomes. Data at preoperative baselines and various intervals during therapy were prospectively and voluntarily provided by 1285 prescribing physicians from 978 centers (911 in the US and Canada and 67 international centers). Neurologists or their designated staff completed standard case report forms based on a patient's medical history or current visit and voluntarily sent these forms to Cyberonics for data entry. At baseline, a patient history and implant form was submitted, containing information on patient demographics, epilepsy etiology and syndrome, historical seizure types and frequencies, quality of life, physician global assessment, and current AEDs. At each follow-up visit, information was collected on seizure types, seizure frequency (overall and by seizure type), a customized quality of life measure, and current AEDs. Active data collection ceased in 2003. Previous investigators have authenticated the integrity of the systems for collecting and processing registry data using an independent auditing agency. ${ }^{2}$ The database was queried in September 2011, and all seizure outcomes reported at $3,6,12$, and 24 months after VNS device implantation were extracted and compared with preoperative baselines using the Engel classification scheme.12 The overall percentage decrease in seizure frequency versus baseline and response rates to VNS therapy were also calculated at each follow-up visit using the seizure rates reported by the treating neurologist at each visit. Patients with a $\geq 50 \%$ decrease in seizure frequency after VNS versus preoperative baseline levels were designated "responders," whereas those without a $\geq 50 \%$ decrease in seizure frquency were labeled "nonresponders." Other data extracted for all patients included sex, race, age at diagnosis, age at stimulator implantation, and preoperative duration of epilepsy.
To determine whether seizure outcomes after VNS therapy differed between patients with or without PTE, epilepsy etiology was examined. Epilepsy etiology was reported by the prescribing physician at the time of device implantation for many patients and was not independently verified. Based on this information, patients were classified into one of the following groups: 1) epilepsy resulting from trauma, or PTE (for example, "posttraumatic," "status post-head injury," "status postcontusion," or "gunshot injury to head"); 2) epilepsy unrelated to trauma, or non-PTE (for example, "mesial temporal sclerosis," "tumor," "infection," or "stroke"); or 3) ambiguous/indeterminate (for example, "possible posttraumatic," "fall," "accident," or "brain hemorrhage"). Patients were excluded from analysis if an ambiguous/indeterminate epilepsy etiology was present, if the etiology was unknown or unreported, if both traumatic and nontraumatic etiologies were reported, if a history of childbirth-related injury was reported (given inability to disaggregate between traumatic, anoxic, or other possible epilepsy etiologies), or if no data from postoperative follow-up evaluations were available. Collected data did not specify the location, severity, or penetrating status of the TBI since the registry was not designed for PTE in particular. Furthermore, the registry is further limited in that there is no information on trauma origin for patients with PTE (that is, military or civilian).

\section{Statistical Analysis}

Patient sex, race, age at diagnosis, age at device implantation, preoperative duration of epilepsy, baseline seizure frequency, baseline seizure type, and number of AEDs were compared for PTE versus non-PTE patients using the Student t-test for continuous variables and the chi-square test for categorical variables. Initial analysis using all available data showed a statistically significant difference in response for PTE versus non-PTE patients, but the 2 groups also had statistical differences in sex and age, suggesting possible confounding by these variables. Thus, a case-control study design was used to control for the potentially influential covariates of sex, age at diagnosis, and age at implantation to assess the association between etiology (PTE versus non-PTE) and percentage of seizure decrease, responder status, or Engel outcome class. To determine if etiology was an independent variable affecting patient outcome, a case-control design was chosen over a multivariate analysis approach, as all of the data for case-control matching were readily available, and the result was a more direct comparison of the outcomes for the 2 groups. During the case-control record selection, for each PTE patient record, 2 non-PTE patient records were matched according to sex, follow-up time, age at implantation, and age at diagnosis. This selection process was done without any consideration for the response variables. Unmatched patient data were removed from analysis. The Wilcoxon rank-sum test and 2-proportion z-test were used to compare the median percentage decrease in seizure frequency and the responder rates, respectively, between PTE and non-PTE patients at each follow-up time point. The Cochran-Mantel-Haenszel statistic for 
row mean scores was used to test whether there was a relationship between etiology type (PTE or non-PTE) and Engel class, controlling for the 4 follow-up time points in all instances. An odds ratio with a $95 \%$ confidence interval was calculated to measure the overall likelihood of attaining a clinical response $(\geq 50 \%$ reduced seizure frequency) between PTE and non-PTE patients by utilizing the Breslow-Day test for homogeneity of the odds ratios at the different follow-up times. The level of significance was set at 0.05 for all analyses. Statistical analysis was performed using SAS version 9.2 (SAS Institute, Inc.).

\section{Results}

Data from patients with PTE or non-PTE etiologies were extracted from 7383 individuals in the VNS patient registry. Patients with unknown, ambiguous, or multiple etiologies, as well as those without postoperative followup, were excluded from analysis (Fig. 1). Overall, data from 637 postoperative visits in 317 PTE patients were compared with 3666 visits in 1763 non-PTE patients.

Initial review of the raw outcome data revealed a progressive decrease in seizure frequency and an increase in the responder rate in both PTE and non-PTE patients over time after VNS therapy. Furthermore, observed trends suggested that, compared with non-PTE patients, the PTE population demonstrated a greater overall decrease in seizure frequency (Fig. 2A), a greater increase in the response rate (Fig. 2B), and more favorable Engel outcomes over time (Fig. 2C-D). However, differences in baseline demographics for the PTE and non-PTE patient pools made unadjusted statistical comparisons inappropriate. As summarized in Table 1, at 24 months after implantation, PTE and non-PTE patient groups exhibited significant differences in sex, age at implant, and age at diagnosis. Thus, a case-control study design was

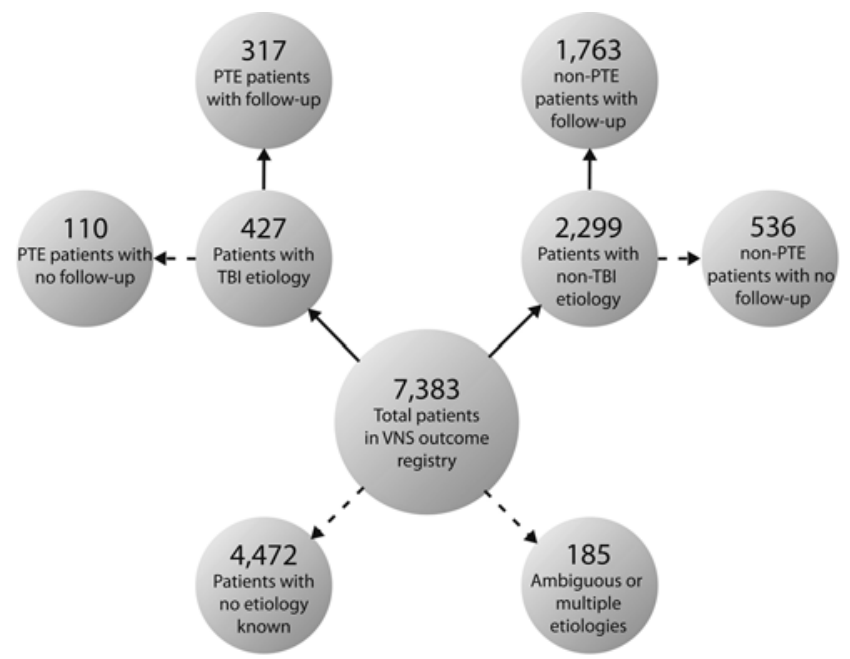

FIG. 1. Schematic of study design summary. Data on patients with PTE or non-PTE were extracted from 7383 individuals in the registry of patients with epilepsy who had received VNS therapy. Data relating to individuals with unknown etiology, ambiguous or multiple etiologies, or those without postoperative follow-up visits were excluded. Solid lines indicate patients included in the analysis; dashed lines, patients excluded from analysis.
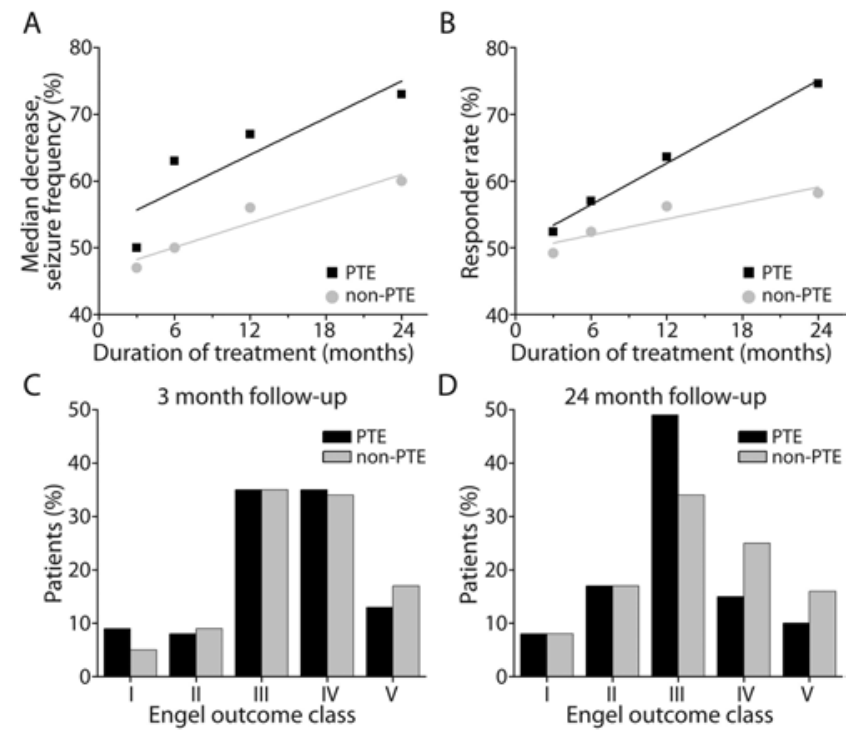

FIG. 2. Graphs demonstrating raw data on seizure outcomes after VNS therapy in PTE versus non-PTE patients. The median percentage decrease in seizure frequency $(A)$ and the responder rates $(B)$ are shown for PTE and non-PTE patients after VNS therapy at 3, 6, 12, and 24 months. A trend toward improved seizure outcomes in PTE versus non-PTE patients is seen over time. Regarding Engel outcomes, little difference is seen between PTE and non-PTE patients at 3 months after VNS therapy (C). But at 24 months after VNS therapy (D), PTE patients demonstrated a more favorable outcome (Engel Class III) more often and a less favorable outcome (Engel Class IV-V) less often, compared with non-PTE patients. For panels A-D, statistical analysis was deferred given the unequal samples (see Table 1). The number of patients with relevant data is $254,158,154$, and 71 for those with PTE and $1449,975,878$, and 364 for those with non-PTE at 3, 6, 12, and 24 months, respectively.

used in which 2 non-PTE patient records were matched to each PTE patient record according to baseline patient demographics without any consideration for the response variables. Repeat analysis of demographic data using the case-control study design revealed no significant differences in baseline characteristics between PTE and nonPTE patients.

Utilizing the case-control study design, a larger progressive reduction in seizure frequency was observed in PTE patients after VNS therapy $(50 \%$ fewer seizures at 3 months; $73 \%$ fewer seizures at 24 months) as compared with non-PTE patients after VNS therapy (46\% fewer seizures at 3 months; $57 \%$ fewer seizures at 24 months; Fig. $3 \mathrm{~A})$. This difference was statistically significant at 6 and 12 months postoperatively ( $\mathrm{p}=0.03$ and $\mathrm{p}<0.01$, respectively) but did not reach statistical significance at 3 or 24 months ( $\mathrm{p}=0.14$ and $\mathrm{p}=0.15$, respectively). Moreover, the rate of clinical response at 24 months was significantly higher among PTE patients (78\% responders) versus non-PTE patients (61\% responders; $p=0.02$; Fig. 3B), leading to more favorable Engel outcomes in the PTE patient population at the last follow-up (Fig. 3C-D). Importantly, the reduction in seizure frequency was greater and the response rate higher in PTE patients versus non-PTE patients at every available follow-up interval analyzed.

Overall, after controlling for the 4 postoperative follow-up durations, there was a strong statistical rela- 
Vagus nerve stimulation for posttraumatic epilepsy

TABLE 1: Summary of demographic data for PTE versus non-PTE patients*

\begin{tabular}{|c|c|c|c|c|}
\hline Variable & $\begin{array}{l}\text { FU Duration } \\
\text { (mos) }\end{array}$ & PTE Patients & Non-PTE Patients & $\mathrm{p}$ Value \\
\hline \multicolumn{5}{|l|}{ raw patient data } \\
\hline $\operatorname{sex}(\% M)$ & 24 & 63 & 50 & $0.04 \ddagger$ \\
\hline \multirow[t]{2}{*}{ race } & 3 & $86 \%$ white; $5 \%$ black; $5 \%$ Hispanic; $4 \%$ other & $83 \%$ white; $5 \%$ black; $7 \%$ Hispanic; $5 \%$ other & 0.39 \\
\hline & 24 & $90 \%$ white; $3 \%$ black; $1 \%$ Hispanic; $6 \%$ other & $85 \%$ white; $3 \%$ black; $4 \%$ Hispanic; $7 \%$ other & 0.59 \\
\hline mean age at implant (yrs) & 24 & $37.2 \pm 13.1$ & $27.5 \pm 14.1$ & $<0.001 \ddagger$ \\
\hline \multirow[t]{2}{*}{ mean age at diagnosis (yrs) } & 3 & $14.5 \pm 14.5$ & $6.8 \pm 10.2$ & $<0.001 \ddagger$ \\
\hline & 24 & $13.5 \pm 10.2$ & $6.8 \pm 10.0$ & $<0.001 \ddagger$ \\
\hline \multirow{2}{*}{$\begin{array}{l}\text { mean preop duration of epi- } \\
\text { lepsy (yrs) }\end{array}$} & 3 & $23.2 \pm 14.1$ & $17.7 \pm 12.6$ & $<0.001 \ddagger$ \\
\hline & 24 & $23.7 \pm 13.0$ & $20.7 \pm 12.7$ & 0.08 \\
\hline \multirow[t]{2}{*}{$\operatorname{sex}(\% M)$} & 3 & 55 & 55 & 0.99 \\
\hline & 24 & 56 & 56 & 0.99 \\
\hline \multirow[t]{2}{*}{ race } & 3 & $87 \%$ white; $5 \%$ black; $4 \%$ Hispanic; $4 \%$ other & $84 \%$ white; $5 \%$ black; $6 \%$ Hispanic; $5 \%$ other & 0.62 \\
\hline & 24 & $88 \%$ white; $3 \%$ black; $2 \%$ Hispanic; $7 \%$ other & $85 \%$ white; $4 \%$ black; $6 \%$ Hispanic; $6 \%$ other & 0.73 \\
\hline \multirow[t]{2}{*}{ mean age at implant (yrs) } & 3 & $37.0 \pm 12.2$ & $35.3 \pm 11.7$ & 0.06 \\
\hline & 24 & $34.2 \pm 12.1$ & $32.8 \pm 10.6$ & 0.45 \\
\hline \multirow[t]{2}{*}{ mean age at diagnosis (yrs) } & 3 & $13.9 \pm 11.2$ & $13.3 \pm 11.3$ & 0.51 \\
\hline & 24 & $11.5 \pm 9.0$ & $10.8 \pm 9.7$ & 0.65 \\
\hline \multirow{2}{*}{$\begin{array}{l}\text { mean preop duration of epi- } \\
\text { lepsy (yrs) }\end{array}$} & 3 & $22.9 \pm 14.0$ & $21.8 \pm 11.2$ & 0.28 \\
\hline & 24 & $22.5 \pm 13.1$ & $21.7 \pm 11.2$ & 0.68 \\
\hline
\end{tabular}

* Values represent the means \pm standard deviations unless indicated otherwise. For raw data, the number of patients with relevant data $=257(\mathrm{PTE})$ and 1468 (non-PTE) at 3 months and 71 (PTE) and 369 (non-PTE) at 24 months. For case-control data, the number of patients with relevant data = 239 (PTE) and 478 (non-PTE) at 3 months and 59 (PTE) and 118 (non-PTE) at 24 months. Abbreviation: FU = follow-up.

$\dagger$ Reflects the Student t-test to compare the means of continuous variables, unless data were nonnormal, in which case the Wilcoxon rank-sum test was used to compare medians (that is, baseline seizure frequency); the chi-square test was used for categorical variables.

‡ Statistically significant value $(p<0.05)$.

$\S$ The median and median absolute deviation are reported.

tionship between etiology (PTE or non-PTE) and Engel class ( $p<0.0001$, Cochran-Mantel-Haenszel statistic), and PTE patients were significantly more likely to attain a favorable clinical response of $\geq 50 \%$ reduced seizure frequency with an OR of 1.32 (95\% CI 1.07-1.61).

Baseline seizure frequency and baseline seizure type were not used as part of the case-control matching process, but looking at these variables after the selections were made revealed that there were no significant differences between the 2 groups after matching at either 3 or 24 months (see Table 1 for seizure frequency and Fig. 4 for seizure types). As many patients presented with multiple seizure types, each seizure type was analyzed separately.

The mean number of AEDs at baseline and at followup visits were compared for PTE versus non-PTE patients (Table 2). For the patients with 3-month data, the nonPTE group had a statistically significantly higher aver- age number of baseline AEDs $(\mathrm{p}=0.02)$, but the actual difference of 2.52 versus 2.36 AEDs would not seem to play a significant role in the observed clinical outcome at 3 months. Among the patients with 24-month data, the difference in baseline AEDs was no longer statistically significant $(p=0.77)$. The number of AEDs taken during the follow-up visits was lower than at baseline for both groups, but the 2 groups no longer showed any statistical difference at 3 or 24 months $(p=0.09$ and $p=0.51$, respectively).

\section{Discussion}

Posttraumatic epilepsy is one of the most challenging forms of epilepsy to treat because of its high rate of resistance to antiepileptic medications and our poor ability to localize epileptic foci for resection. ${ }^{15}$ Given the 500,000 
A

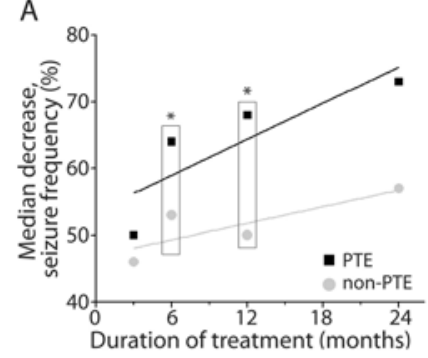

C

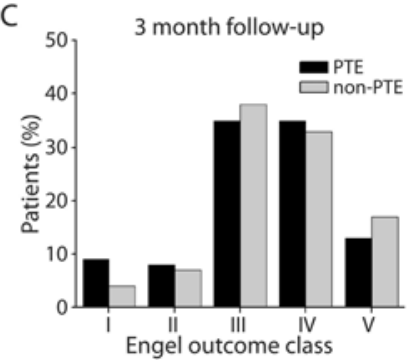

B

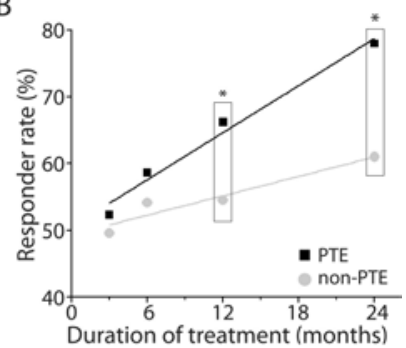

D

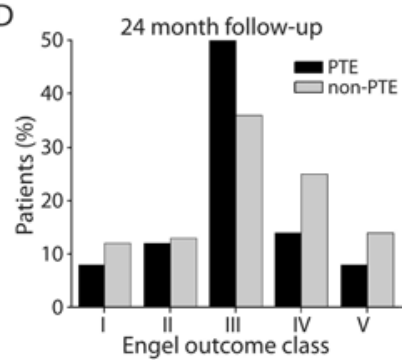

FIG. 3. Graphs demonstrating seizure outcomes after VNS therapy in PTE versus non-PTE patients utilizing a case-control study design. The median percentage decrease in seizure frequency $(\mathbf{A})$ and the responder rates (B) are shown for PTE and non-PTE patients after VNS therapy at $3,6,12$, and 24 months. For the decrease in seizure frequency, differences were statistically significant at 6 and 12 months ( $p=$ 0.03 and $p<0.01$, respectively) but not at 3 or 24 months ( $p=0.14$ and $p$ $=0.15$, respectively). For responder rates, differences were statistically significant at 12 and 24 months ( $p=0.03$ and $p=0.02$, respectively) but not at 3 or 6 months ( $p=0.49$ and $p=0.38$, respectively). Regarding Engel outcomes at 3 (C) and 24 (D) months after VNS therapy, little difference is seen between PTE and non-PTE patients at 3 months. But at 24 months after VNS therapy, PTE patients demonstrated a more favorable outcome (Engel Class III) more often and a less favorable outcome (Engel Class IV-V) less often, compared with non-PTE patients $(p<0.0001)$. For panels $A$ and $B$, statistical analysis reflects the Wilcoxon rank-sum test and 2-proportion z-test, respectively. For $\mathrm{C}$ and $D$, statistical analysis reflects the Cochran-Mantel-Haenszel statistic for row mean scores. Asterisk indicates statistically significant difference $(p<0.05)$. The number of patients with relevant data is $239,145,133$, and 59 for those with PTE and 478, 290, 266, and 118 for those with non-PTE at 3, 6, 12, and 24 months, respectively.

yearly admissions to US hospitals for TBI, there is a dire need for improved treatment strategies for PTE. Here, we analyzed a large, prospectively collected registry of patients with medically refractory epilepsy who had received VNS therapy, and we compared seizure outcomes in patients with PTE versus non-PTE using a case-control study design. The specific type of injury was not specified for the registry data, so the analysis applies to PTE in general without data regarding specific subtypes. Our results suggest that over time patients with PTE receive increased benefit from VNS therapy as compared with patients with nontraumatic epilepsy etiologies, including a greater decrease in seizure frequency and a more favorable Engel outcome class. The median decrease in seizure frequency of $73 \%$ at 24 months in PTE patients was not statistically different from the $57 \%$ decrease in non-PTE patients, and it is unknown if this difference would be clinically significant for the typical patient. However, 78\% of PTE patients responded favorably (that is, $\geq 50 \%$ reduction in seizure frequency) after 24 months of treatment, as compared with only $61 \%$ of non-PTE

patients (OR 1.32, 95\% CI 1.07-1.61). This difference is important, as practitioners may rely on responder rates in determining whether to treat with VNS therapy. Overall, these findings are in line with recent observations, including those in 2 retrospective cases series ${ }^{11,24}$ and 1 meta-analysis ${ }^{14}$ suggesting that PTE patients attain even greater clinical benefit from VNS therapy than patients with drug-resistant epilepsy unrelated to trauma.

What are the neurobiological mechanisms of VNS in the treatment of PTE? Previous human and animal studies have demonstrated that vagal stimulation leads to desynchronization and an overall decrease in abnormal electroencephalographic spiking patterns, which may contribute to its antiepileptogenic effects..$^{10,18,23}$ Recently, Smith and colleagues ${ }^{33}$ specifically examined neurophysiological changes induced by VNS in a fluid percussion injury model of PTE in animals. These authors observed psychomotor recovery in brain-injured rats after vagal stimulation, suggesting enhanced neural plasticity related to norepinephrine release, as well as increased survival of neurons secreting $\gamma$-aminobutyric acid. ${ }^{27}$ While further basic research is necessary to fully elucidate these mechanisms, it is possible that VNS triggers remodeling of the neural circuitry, including the preservation of inhibitory neurotransmission in patients with TBI, contributing to the clinical benefit in patients with PTE.

It is important to recognize the limitations of VNS therapy in PTE and other epilepsies to guide treatment discussions and manage the expectations of both patients and physicians. While many patients with epilepsy benefit from VNS, complete seizure freedom is rare, and some patients unfortunately experience no improvement in symptoms. Additionally, it is important to appreciate the often delayed clinical benefit of VNS during the first few months of its application. Previous studies have shown that VNS improves quality of life and mood in patients with epilepsy. ${ }^{3,22}$ And, in epilepsy in general, there is some evidence linking seizure frequency with quality of life. ${ }^{26,34}$ However, other authors dispute that there is any significant quality-of-life improvement when there is anything less than complete seizure freedom.,25 Further study on quality-of-life measures in VNS therapy is needed, but given the known debilitating effects of frequent seizures, VNS may be considered in patients with PTE who are poor candidates for resection and in whom medical therapy has failed.

Besides patients with epilepsy, those with TBI suffer from other neurological and psychological sequelae not studied in this report, including depression, anxiety disorders, neurocognitive deficits, and chronic headache..$^{19,28}$ Vagus nerve stimulation has provided benefit in patients suffering from treatment-resistant depression ${ }^{16,29,30}$ and received FDA approval for this therapeutic purpose in 2005. Possible therapeutic effects of VNS in drug-resistant anxiety disorders and chronic headache are also being examined..$^{5,8,17,20}$ Thus, the potential effects of VNS on neuropsychological profiles in patients with TBI will be important to consider going forward.

It is necessary to acknowledge significant limitations in the present study. First, registry data were derived from an industry-sponsored database and analyzed in part by 
Vagus nerve stimulation for posttraumatic epilepsy

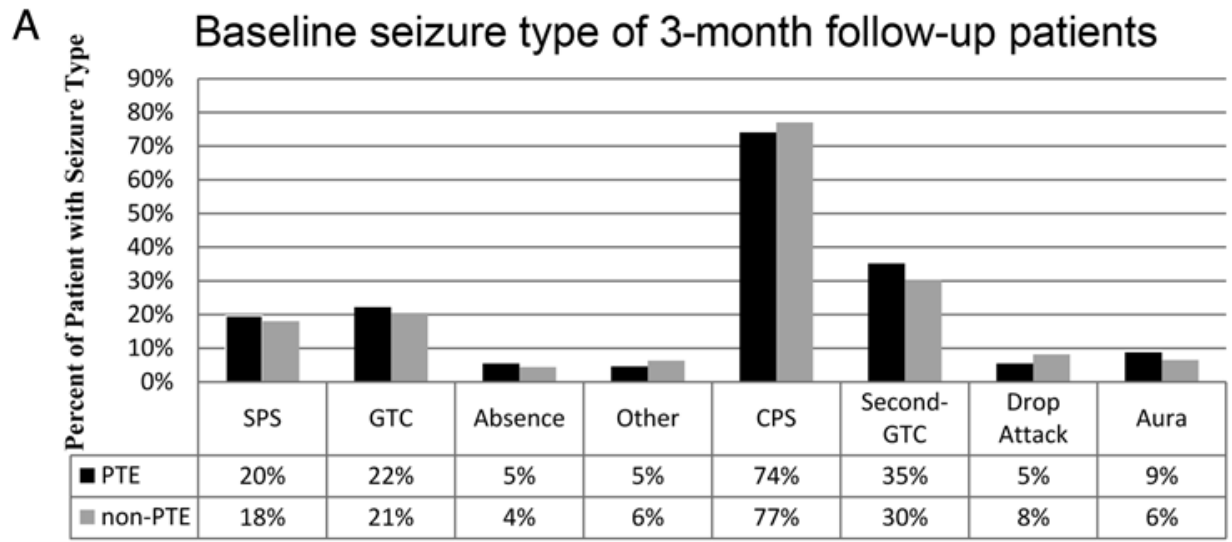

\begin{tabular}{|c|c|c|c|c|c|c|c|c|}
\hline 3-month & SPS & GTC & Absence & Other & CPS & Second-GTC & Drop Attack & Aura \\
\hline PTE & 47 & 53 & 13 & 11 & 177 & 84 & 13 & 21 \\
\hline non-PTE & 86 & 98 & 21 & 30 & 368 & 144 & 39 & 31 \\
\hline p-value & 0.59 & 0.60 & 0.53 & 0.36 & 0.39 & 0.17 & 0.19 & 0.26 \\
\hline
\end{tabular}

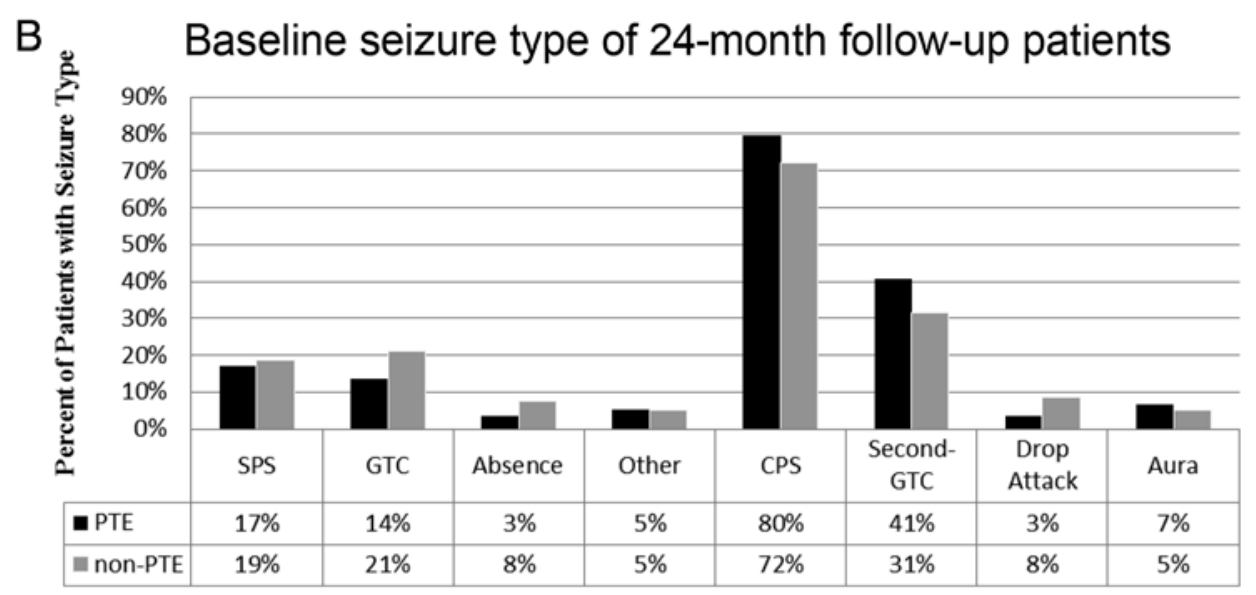

\begin{tabular}{|c|c|c|c|c|c|c|c|c|}
\hline 24-month & SPS & GTC & Absence & Other & CPS & Second-GTC & Drop Attack & Aura \\
\hline PTE & 10 & 8 & 2 & 3 & 47 & 24 & 2 & 4 \\
\hline non-PTE & 22 & 25 & 9 & 6 & 85 & 37 & 10 & 6 \\
\hline p-value & 0.78 & 0.22 & 0.27 & 0.99 & 0.27 & 0.22 & 0.20 & 0.64 \\
\hline
\end{tabular}

FIG. 4. Graphs depicting baseline seizure types for 3-month $(\mathbf{A})$ and 24-month $(B)$ follow-up patients with comparison statistics. CPS = complex partial seizure; GTC = generalized tonic-clonic seizure; second-GTC = secondarily GTC; SPS = simple partial seizure.

Cyberonics, Inc., the manufacturer of the VNS therapy system. This conflict of interest raises the issue of potential bias, but it is also important to note that previous investigators have authenticated the integrity of the systems for collecting and processing the registry data by using an independent auditing agency. ${ }^{3}$ Next, given the nature of patient registries, we cannot independently ascertain the validity of clinical data submitted by individual phy-

TABLE 2: Case-control data: AEDs of PTE versus non-PTE patients*

\begin{tabular}{ccccc}
\hline Variable & FU Duration (mos) & PTE Patients & Non-PTE Patients & $p$ Value \\
\hline baseline AEDs & 3 & $2.36 \pm 0.92$ & $2.52 \pm 0.89$ & $0.02 \ddagger$ \\
AEDs at FU & 24 & $2.32 \pm 1.01$ & $2.36 \pm 0.92$ & 0.77 \\
& 3 & $2.10 \pm 1.13$ & $2.26 \pm 1.17$ & 0.09 \\
\hline
\end{tabular}

* The number of patients $=239$ (PTE) and 478 (non-PTE) at 3 months and 59 (PTE) and 118 (non-PTE) at 24 months.

$\dagger$ Reflects the Student t-test to compare the means.

$\ddagger$ Statistically significant value $(p<0.05)$. 
sicians. Importantly, epilepsy etiology, as analyzed here as posttraumatic or nontraumatic, was not independently verified, and thus we are relying on the accurate reporting of the prescribing physicians. While measures were taken to enhance the reliability of our results, such as the exclusion of ambiguous epilepsy etiologies and the utilization of a case-control study design, the possibilities of reporting inaccuracy and selection bias cannot be excluded. Clearly, this uncertainty would be best addressed in a large prospective trial of VNS therapy in patients with PTE. Regardless, the strength of this evaluation lies in the ability to pool a very large number of cases, which would be difficult to achieve even in a multiinstitutional trial.

\section{Conclusions}

There is a great need for improved treatment strategies for PTE, as it is one of the most challenging forms of epilepsy to manage. Vagus nerve stimulation is an adjunctive treatment for patients with medically refractory epilepsy who are not good candidates for definitive resection. Utilizing a case-control study design to analyze seizure outcomes from a large, prospectively collected patient registry, we found that patients with PTE may achieve better outcomes after VNS therapy than individuals with nontraumatic epilepsy etiologies. Overall, $78 \%$ of patients with PTE responded favorably to VNS therapy (that is, $\geq 50 \%$ reduction in seizure frequency) after 24 months of treatment, compared with only $61 \%$ of patients with non-PTE (OR 1.32, 95\% CI 1.07-1.61). These data suggest that VNS may be considered in patients with medically refractory PTE who are not favorable candidates for resection. A controlled prospective study is necessary to validate these observations.

\section{Disclosure}

Two authors (K.H.H. and C.M.G.) are employees of Cyberonics, Inc., the manufacturer of the VNS Therapy System and sponsor of the VNS Therapy Patient Outcome Registry. They assisted in the statistical analyses.

Author contributions to the study and manuscript preparation include the following. Conception and design: Englot, Chang. Acquisition of data: Gordon. Analysis and interpretation of data: Englot, Rolston, Wang, Chang. Drafting the article: Englot. Critically revising the article: all authors. Reviewed submitted version of manuscript: all authors. Approved the final version of the manuscript on behalf of all authors: Englot. Statistical analysis: Englot, Hassnain, Gordon. Study supervision: Chang.

\section{References}

1. Agrawal A, Timothy J, Pandit L, Manju M: Post-traumatic epilepsy: an overview. Clin Neurol Neurosurg 108:433-439, 2006

2. Amar AP, Apuzzo ML, Liu CY: Vagus nerve stimulation therapy after failed cranial surgery for intractable epilepsy: results from the vagus nerve stimulation therapy patient outcome registry. Neurosurgery 55:1086-1093, 2004

3. Amar AP, Apuzzo ML, Liu CY: Vagus nerve stimulation therapy after failed cranial surgery for intractable epilepsy: results from the vagus nerve stimulation therapy patient outcome registry. Neurosurgery 62 (Suppl 2):506-513, 2008

4. Annegers JF, Coan SP: The risks of epilepsy after traumatic brain injury. Seizure 9:453-457, 2000
5. Ansari S, Chaudhri K, Al Moutaery KA: Vagus nerve stimulation: indications and limitations. Acta Neurochir Suppl (Wien) 97:281-286, 2007

6. Ben-Menachem E: Vagus-nerve stimulation for the treatment of epilepsy. Lancet Neurol 1:477-482, 2002

7. Birbeck GL, Hays RD, Cui X, Vickrey BG: Seizure reduction and quality of life improvements in people with epilepsy. Epilepsia 43:535-538, 2002

8. Cecchini AP, Mea E, Tullo V, Curone M, Franzini A, Broggi G, et al: Vagus nerve stimulation in drug-resistant daily chronic migraine with depression: preliminary data. Neurol Sci 30 (Suppl 1):S101-S104, 2009

9. Chang EF, Barbaro NM: Nonresective epilepsy surgery. Epilepsia 51 (Suppl 1):87-89, 2010

10. Chase MH, Nakamura Y, Clemente CD, Sterman MB: Afferent vagal stimulation: neurographic correlates of induced EEG synchronization and desynchronization. Brain Res 5:236-249, 1967

11. Elliott RE, Morsi A, Kalhorn SP, Marcus J, Sellin J, Kang M, et al: Vagus nerve stimulation in 436 consecutive patients with treatment-resistant epilepsy: long-term outcomes and predictors of response. Epilepsy Behav 20:57-63, 2011

12. Engel J Jr (ed): Outcome with respect to epileptic seizures, in Surgical Treatment of the Epilepsies. New York: Raven Press, 1987, pp 553-572

13. Englot DJ, Chang EF, Auguste KI: Efficacy of vagus nerve stimulation for epilepsy by patient age, epilepsy duration, and seizure type. Neurosurg Clin N Am 22:443-448, v, 2011

14. Englot DJ, Chang EF, Auguste KI: Vagus nerve stimulation for epilepsy: a meta-analysis of efficacy and predictors of response. A review. J Neurosurg 115:1248-1255, 2011

15. Garga N, Lowenstein DH: Posttraumatic epilepsy: a major problem in desperate need of major advances. Epilepsy Curr 6:1-5, 2006

16. George MS, Rush AJ, Marangell LB, Sackeim HA, Brannan SK, Davis SM, et al: A one-year comparison of vagus nerve stimulation with treatment as usual for treatment-resistant depression. Biol Psychiatry 58:364-373, 2005

17. George MS, Ward HE Jr, Ninan PT, Pollack M, Nahas Z, Anderson B, et al: A pilot study of vagus nerve stimulation (VNS) for treatment-resistant anxiety disorders. Brain Stimulat 1:112121,2008

18. Henry TR: Therapeutic mechanisms of vagus nerve stimulation. Neurology 59 (6 Suppl 4):S3-S14, 2002

19. Hoffman JM, Lucas S, Dikmen S, Braden CA, Brown AW, Brunner R, et al: Natural history of headache after traumatic brain injury. J Neurotrauma 28:1719-1725, 2011

20. Hord ED, Evans MS, Mueed S, Adamolekun B, Naritoku DK: The effect of vagus nerve stimulation on migraines. J Pain 4: 530-534, 2003

21. Jabbari B, Prokhorenko O, Khajavi K, Mena H: Intractable epilepsy and mild brain injury: incidence, pathology and surgical outcome. Brain Inj 16:463-467, 2002

22. Klinkenberg S, Majoie HJ, van der Heijden MM, Rijkers K, Leenen L, Aldenkamp AP: Vagus nerve stimulation has a positive effect on mood in patients with refractory epilepsy. Clin Neurol Neurosurg 114:336-340, 2012

23. Koo B: EEG changes with vagus nerve stimulation. J Clin Neurophysiol 18:434-441, 2001

24. Lee HO, Koh EJ, Oh YM, Park SS, Kwon KH, Choi HY: Effect of vagus nerve stimulation in post-traumatic epilepsy and failed epilepsy surgery: preliminary report. J Korean Neurosurg Soc 44:196-198, 2008

25. Luoni C, Bisulli F, Canevini MP, De Sarro G, Fattore C, Galimberti CA, et al: Determinants of health-related quality of life in pharmacoresistant epilepsy: results from a large multicenter study of consecutively enrolled patients using validated quantitative assessments. Epilepsia 52:2181-2191, 2011

26. McLachlan RS, Rose KJ, Derry PA, Bonnar C, Blume WT, 
Girvin JP: Health-related quality of life and seizure control in temporal lobe epilepsy. Ann Neurol 41:482-489, 1997

27. Neese SL, Sherill LK, Tan AA, Roosevelt RW, Browning RA, Smith DC, et al: Vagus nerve stimulation may protect GABAergic neurons following traumatic brain injury in rats: an immunocytochemical study. Brain Res 1128:157-163, 2007

28. Riggio S: Traumatic brain injury and its neurobehavioral sequelae. Psychiatr Clin North Am 33:807-819, 2010

29. Rush AJ, George MS, Sackeim HA, Marangell LB, Husain MM, Giller C, et al: Vagus nerve stimulation (VNS) for treatment-resistant depressions: a multicenter study. Biol Psychiatry 47:276-286, 2000

30. Rush AJ, Sackeim HA, Marangell LB, George MS, Brannan SK, Davis SM, et al: Effects of 12 months of vagus nerve stimulation in treatment-resistant depression: a naturalistic study. Biol Psychiatry 58:355-363, 2005

31. Salazar AM, Jabbari B, Vance SC, Grafman J, Amin D, Dillon JD: Epilepsy after penetrating head injury. I. Clinical correlates: a report of the Vietnam Head Injury Study. Neurology 35:1406-1414, 1985
32. Schachter SC, Saper CB: Vagus nerve stimulation. Epilepsia 39:677-686, 1998

33. Smith DC, Modglin AA, Roosevelt RW, Neese SL, Jensen RA, Browning RA, et al: Electrical stimulation of the vagus nerve enhances cognitive and motor recovery following moderate fluid percussion injury in the rat. J Neurotrauma 22: 1485-1502,2005

34. von Lehe M, Lutz M, Kral T, Schramm J, Elger CE, Clusmann $\mathrm{H}$ : Correlation of health-related quality of life after surgery for mesial temporal lobe epilepsy with two seizure outcome scales. Epilepsy Behav 9:73-82, 2006

Manuscript submitted January 1, 2012.

Accepted August 15, 2012.

Please include this information when citing this paper: published online September 14, 2012; DOI: 10.3171/2012.8.JNS122.

Address correspondence to: Dario J. Englot, M.D., Ph.D., Department of Neurological Surgery, University of California, San Francisco, 505 Parnassus Avenue, Box 0112, San Francisco, California 94143-0112. email: EnglotDJ@ neurosurg.ucsf.edu. 